\title{
Avaliação comparativa do ritmo de piscar em crianças normais em idade pré-escolar
}

\author{
Comparative evaluation of healthy preschool children blink
}

\author{
Marcelo Mendes Lavezzo ${ }^{1}$ \\ Silvana Artioli Schellini ${ }^{2}$ \\ Carlos Roberto Padovani ${ }^{3}$
}

\section{RESUMO}

Objetivo: Estabelecer o padrão de normalidade do ritmo de piscar em crianças normais em idade pré-escolar. Métodos: Avaliaram-se 200 crianças de 4 a 6 anos, saudáveis, usando tomada de imagens digitais, nos planos frontal e lateral, em estado de vigília, em posição primária do olhar, estando o objeto de observação localizado na altura da pupila. Para a tomada das imagens foi utilizada uma filmadora Sony Lithium, sendo as mesmas gravadas em fitas $8 \mathrm{~mm}$, transferidas para um computador MacIntosh G4 e processadas pelo programa iMovie, estudando-se: o tempo de abertura e o tempo de fechamento palpebral, o tempo de piscar completo e o ritmo de movimentos palpebrais por minuto, durante 3 minutos. Os resultados foram avaliados por estatística descritiva e gráfico de linhas. Resultados: O piscar completo foi mais freqüente que o incompleto. O ritmo do piscar completo aumenta com o aumento da idade. Para o piscar incompleto, os valores foram semelhantes em todas as idades avaliadas. $\mathrm{O}$ tempo de fechamento e de abertura palpebral e o tempo de piscar completo foram semelhantes em meninos e meninas. $\mathrm{O}$ tempo de fechamento foi mais lento que o tempo de abertura palpebral. Conclusões: $O$ ritmo de piscar completo aumenta com a idade. Os tempos de fechamento e de abertura palpebral e o tempo de piscar completo foram semelhantes em ambos os sexos, em todas as idades estudadas, sendo o fechamento mais lento que a abertura palpebral.

Descritores: Piscadela/fisiologia; Movimentos sacádicos; Pálpebras; Gravação de videoteipe; Pré-escolar

\section{INTRODUÇ̃̃̃O}

O piscar tem várias funções, tais como: impedir a dessecação, eliminar corpos estranhos, excluir estímulos visuais, distribuir e manter o filme lacrimal $^{(1)}$.

O ritmo de piscar varia de acordo com muitos fatores, sendo, em adultos, diferente nas diversas faixas etárias ${ }^{(2)}$.

O movimento de piscar ocorre graças à ação antagonista entre os músculos orbicular do olho e o levantador da pálpebra superior ${ }^{(3-5)}$. A rápida ativação dos motoneurônios do músculo orbicular, juntamente com a breve inibição da atividade tônica dos motoneurônios do músculo levantador da pálpebra superior, conduzem ao fechamento palpebral - a fase de fechamento do piscar. A atividade rápida do levantador da pálpebra superior, imediatamente após o declínio da ativação do orbicular, assegura a reabertura palpebral - a fase de abertura palpebral ${ }^{(5-7)}$.

Assim, as características das duas fases do piscar são produto de padrões de descarga diferencial dos motoneurônios, composição do tipo 
morfológico da fibra muscular e mecanismos passivos da maquinaria palpebral ${ }^{(6-9)}$.

Há dois tipos de piscar: o involuntário ou reflexo (que ocorre espontaneamente) e o voluntário (que depende da vontade do paciente). O piscar involuntário é dividido em piscar espontâneo, que ocorre, aproximadamente, em intervalos constantes, e em reflexo de piscar involuntário de medo, que ocorre em resposta a um estímulo externo da córnea ${ }^{(1)}$. O piscar involuntário dura de 290 a 750 milissegundos, sendo influenciado por inúmeras condições, como luminosidade local, temperatura, velocidade das correntes de ar, patologias oculares e pelo nível de atenção ${ }^{(10)}$.

$\mathrm{O}$ ritmo de piscar influencia a drenagem lacrimal ${ }^{(11)}$ e a integridade da superfície ocular ${ }^{(12)}$ e é influenciado por várias condições, dentre elas: o horário do dia - ritmo aumentado à noite $^{(13)}$; o sexo - homens têm maior ritmo que mulheres ${ }^{(14)}$; nível de atenção ${ }^{(11)}$ e existência de alterações oculares ${ }^{(10)}$.

Por causa da influência de muitos fatores sobre o ritmo de piscar, a avaliação do mesmo é difícil de ser feita.

Em adultos normais, o ritmo de piscar é, em média, de 2 a 50 piscadas por minuto ${ }^{(15)}$, sendo maior o tempo gasto para o fechamento das pálpebras do que para sua abertura, em ambos os sexos, com tendência a tempo maior para fechamento palpebral nas faixas etárias mais elevadas ${ }^{(2)}$.

Utilizando o programa iMovie, é possível detectar alterações da ordem de 1/30 de segundo ( 1 frame), o que se faz necessário, uma vez que a velocidade do movimento palpebral é bastante elevada, estimada em $14 \mathrm{~cm} /$ segundo $^{(1)}$.

Apesar da afirmação de que o ritmo de piscar é mais influenciado por processos cognitivos do que pela idade, cor dos olhos ou fatores locais ${ }^{(14)}$, pouco é descrito em relação ao ritmo de piscar de crianças normais. Sabe-se que crianças com hiperatividade e deficiência de atenção possuem ritmo de piscar aumentado em comparação com crianças normais ${ }^{(16)}$.

Uma das possíveis razões para o não estudo do ritmo de piscar em crianças poderia ser a dificuldade de avaliar os movimentos palpebrais sem que se interfira no mecanismo involuntário do piscar. A introdução de técnicas de gravação e processamento de imagens digitais pode permitir uma melhor avaliação do ritmo de piscar em crianças. O objetivo deste estudo é avaliar a dinâmica palpebral de crianças normais de 4 a 6 anos, usando o sistema de imagens digitais, com o intuito de estabelecer um padrão de resposta para crianças normais desta faixa etária.

\section{MÉTODOS}

Foram avaliadas prospectivamente 200 crianças normais, em idade pré-escolar, de 4 a 6 anos, que freqüentam o Colégio La Salle ou a CEPLASB, da cidade de Botucatu (SP).

Os critérios de inclusão foram: crianças sadias, sem doenças ou cirurgias (oculares ou palpebrais) prévias. Foram excluídas as crianças cujos pais ou responsáveis não forneceram autorização para a realização da pesquisa.
A avaliação foi feita com tomada de imagens digitais, nos planos frontal e lateral, em estado de vigília, em posição primária do olhar, estando o objeto de observação localizado na altura da pupila.

Todas as imagens foram tomadas, sempre pelo mesmo observador, na mesma sala, localizada nas dependências do Colégio La Salle ou na CEPLASB, sob as mesmas condições atmosféricas e de iluminação.

Para a tomada das imagens foi utilizada uma filmadora Sony Lithium, sendo que as mesmas gravadas em fitas $8 \mathrm{~mm}$, transferidas para um computador MacIntosh G4 e processadas pelo programa iMovie.

A dinâmica palpebral foi avaliada tomando-se imagens do olhar, enquanto a criança fixava um objeto localizado a cerca de um metro de distância, durante 3 minutos, com foco no piscar espontâneo, estudando-se: o tempo de abertura palpebral, o tempo de fechamento palpebral, o tempo de piscar completo e o número (ritmo) de movimentos palpebrais por minuto, com avaliação das imagens dinâmicas durante três minutos, seguindo-se a mesma metodologia empregada por alguns autores ${ }^{(2)}$.

Os resultados obtidos foram submetidos a estudo estatístico e estão apresentados segundo métodos de Estatística Descritiva (média, desvio padrão, mediana e quartis) e gráfico de linhas, segundo variável estudada e idade de observação.

\section{RESULTADOS}

O piscar completo foi mais freqüente que o incompleto, com média de 23,84 e 18,98 respectivamente. Os tempos de fechamento palpebral, de abertura e o do piscar completo foram semelhantes, nos 3 momentos de observação. O ritmo de piscar completo foi de cerca de 6 a 23 movimentos por minuto. Não houve diferença estatística significativa em nenhum destes parâmetros estudados (Tabela 1).

Comparando-se meninos e meninas, não houve diferença entre os sexos, tanto com relação ao piscar completo, como incompleto (Tabela 2).

Aos 4 anos o ritmo de piscar completo foi menor que aos 5 e este foi menor que aos 6 anos, com valores de desvio padrão também crescentes nesta ordem. Para o piscar incompleto, os valores foram semelhantes em todas as idades (Tabela 3 ).

O tempo de fechamento foi semelhante em meninos e meninas, para todos os momentos de observação. O mesmo observou-se com relação ao tempo de abertura palpebral e ao tempo de piscar completo. Comparando-se o tempo de fechamento com o tempo de abertura palpebral, o tempo de abertura foi discretamente inferior ao tempo de fechamento, ou seja, o fechamento é mais lento que a abertura palpebral (Tabela 4).

O tempo de fechamento palpebral foi semelhante para todas as idades estudadas, em todos os minutos de observação. O mesmo pode se dizer do tempo de abertura e de piscar completo, quando não houve diferença entre as idades estudadas (Tabela 5). 


\begin{tabular}{|c|c|c|c|c|c|c|c|}
\hline Variável & № de indivíduos & Valor mínimo & Mediana & Valor máximo & Média & Desvio-padrão & \\
\hline Piscar completo & 200 & 0 & 17 & 193 & 23,86 & 24,78 & \\
\hline Piscar incompleto & 200 & 2 & 16 & 60 & 18,98 & 12,04 & \\
\hline TFP - $1^{\circ}$ minuto & 191 & 3 & 7 & 15 & 6,80 & 2,03 & \\
\hline TFP - $2^{\circ}$ minuto & 194 & 3 & 7 & 19 & 7,01 & 2,01 & $p>0,05$ \\
\hline TFP - $3^{\circ}$ minuto & 195 & 4 & 7 & 19 & 6,97 & 2,11 & \\
\hline TAP - $1^{\circ}$ minuto & 191 & 3 & 6 & 11 & 5,84 & 1,45 & \\
\hline TAP - $2^{\circ}$ minuto & 194 & 3 & 6 & 10 & 5,98 & 1,39 & $p>0,05$ \\
\hline TAP - $3^{\circ}$ minuto & 195 & 3 & 6 & 12 & 5,99 & 1,53 & \\
\hline TPC - $1^{\circ}$ minuto & 191 & 6 & 12 & 23 & 12,64 & 3,22 & \\
\hline TPC - $2^{\circ}$ minuto & 194 & 7 & 13 & 24 & 12,99 & 3,09 & $p>0,05$ \\
\hline TPC - $3^{\circ}$ minuto & 195 & 7 & 13 & 30 & 12,96 & 3,37 & \\
\hline
\end{tabular}

\begin{tabular}{|c|c|c|c|}
\hline Sexo & $\begin{array}{l}\text { Medida } \\
\text { descritiva }\end{array}$ & $\begin{array}{c}\text { Ritmo } \\
\text { completo }\end{array}$ & $\begin{array}{c}\text { Ritmo } \\
\text { incompleto }\end{array}$ \\
\hline \multirow{6}{*}{ Masculino } & $\mathrm{N}$ & 98 & 98 \\
\hline & $\mathrm{Vm}$ & 0 & 2 \\
\hline & $\mathrm{Me}$ & 16 & 17 \\
\hline & VM & 161 & 60 \\
\hline & $x$ & 23,63 & 19,37 \\
\hline & S & 23,89 & 10,81 \\
\hline \multirow{6}{*}{ Feminino } & $\mathrm{N}$ & 102 & 102 \\
\hline & $\mathrm{Vm}$ & 1 & 2 \\
\hline & $\mathrm{Me}$ & 17 & 14 \\
\hline & VM & 193 & 99 \\
\hline & $x$ & 24,08 & 18,60 \\
\hline & $\mathrm{S}$ & 25,72 & 13,17 \\
\hline \multicolumn{4}{|c|}{$\begin{array}{l}\qquad p>0,05(M=F) \quad p>0,05(M=F) \\
N=\text { Número de movimentos palpebrais; } V m=\text { Valor mínimo; } M e=\text { Mediana; } V M= \\
\text { Valor máximo; } X=\text { Média; } S=\text { Desvio-padrão; } M=\text { Masculino; } F=\text { Feminino }\end{array}$} \\
\hline
\end{tabular}

\section{DISCUSSÃO}

A avaliação do ritmo de piscar em crianças dificilmente pode ser feita, sendo necessário se adotar parâmetros padronizados, além de se ter a influência direta da colaboração dos participantes, nem sempre fácil na faixa etária pediátrica.

O comportamento da criança influencia no nível de resposta que se pode obter.

É muito importante controlar as variáveis que podem interferir com os resultados, uma vez que o ritmo do piscar espontâneo sofre a influência de muitas variáveis, tanto internas (nível de concentração, humor), como externas (poeira, vento, fumaça) ${ }^{(15)}$.

Também as condições da superfície ocular podem alterar o ritmo de piscar, sendo muito importante se ter como critério de inclusão, a avaliação de indivíduos sem doenças oculares ou palpebrais $^{(12,17)}$.

Como o piscar sofre a influência do comportamento, sendo menor durante a leitura e aumentando durante o diálogo ${ }^{(14)}$, decidiu-se no presente trabalho avaliar as crianças durante

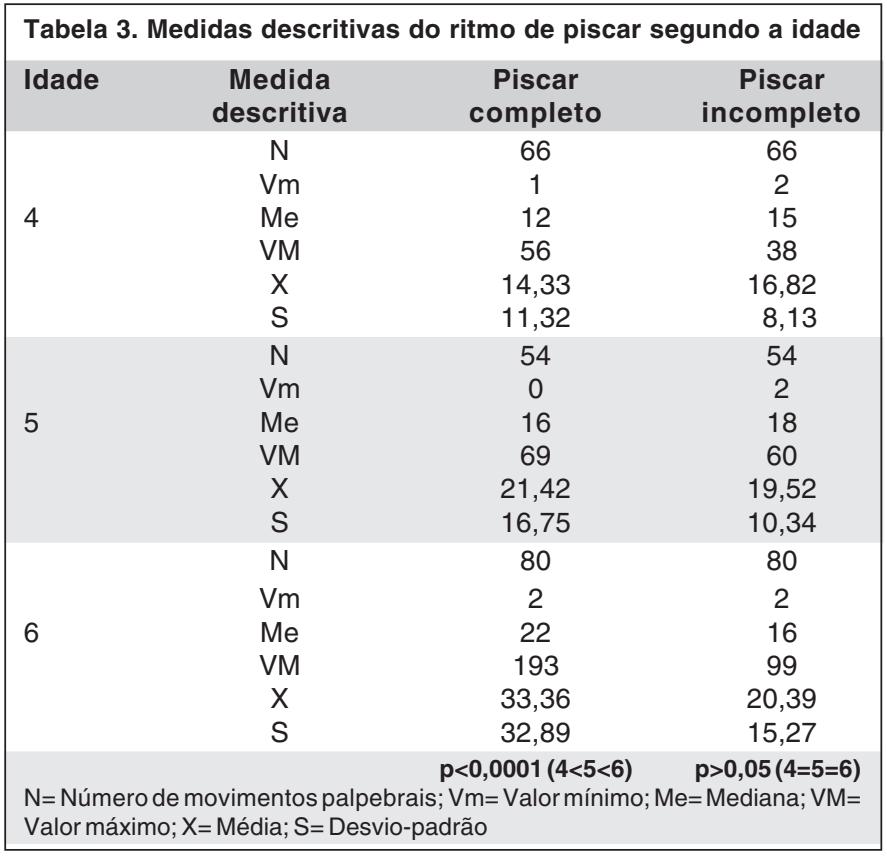

três minutos, tendo-se o primeiro minuto como piscar com atenção, momento em que a criança toma contacto com a filmadora e com o examinador, e, nos demais, a possibilidade de manifestação realmente do piscar espontâneo. Uma vez adaptada ao exame, a criança se distrai, podendo-se supor ser a medida principalmente do terceiro minuto a mais fidedigna do piscar espontâneo. Outra medida que se adotou para tirar a concentração e que permitiu avaliar melhor o piscar espontâneo foi a filmagem na lateral da face da criança. Porém, não houve diferença entre as avaliações feitas nos três minutos de observação.

Quanto aos valores observados, observou-se que o ritmo de piscar completo foi superior ao do incompleto.

O número de piscadas em crianças variou de 6 a 23 ou 30 movimentos por minuto, tendo sido relatado como 2 a 50 piscadas por minuto em adultos ${ }^{(15)}$. 


\begin{tabular}{|c|c|c|c|c|c|}
\hline \multirow[t]{2}{*}{ Variável } & \multirow[t]{2}{*}{ Sexo } & \multirow{2}{*}{$\begin{array}{l}\text { Medida } \\
\text { descritiva }\end{array}$} & \multicolumn{3}{|c|}{ Momento } \\
\hline & & & 10 Minuto & 20 Minuto & 3은 Minuto \\
\hline \multirow{12}{*}{ TFP } & \multirow{6}{*}{ Masculino } & $\mathrm{N}$ & 91 & 94 & 95 \\
\hline & & Vm & 3 & 3 & 4 \\
\hline & & $\mathrm{Me}$ & 7 & 7 & 7 \\
\hline & & VM & 12 & 14 & 19 \\
\hline & & $\mathrm{X}$ & 6,57 & 6,73 & 6,84 \\
\hline & & $\mathrm{S}$ & 1,79 & 1,84 & 2,16 \\
\hline & \multirow{6}{*}{ Feminino } & $\mathrm{N}$ & 100 & 100 & 100 \\
\hline & & $\mathrm{Vm}$ & 3 & 3 & 4 \\
\hline & & $\mathrm{Me}$ & 7 & 7 & 7 \\
\hline & & VM & 15 & 19 & 15 \\
\hline & & $x$ & $7,01 \quad(\mathrm{M}=\mathrm{F})$ & $7,26(\mathrm{M}=\mathrm{F})$ & $7,10 \quad(\mathrm{M}=\mathrm{F})$ \\
\hline & & $S$ & $2,21 \quad p>0,05$ & $2,13 p>0,05$ & $2,07 \quad p>0,05$ \\
\hline \multirow{12}{*}{ TAP } & \multirow{6}{*}{ Masculino } & $\mathrm{N}$ & 91 & 94 & 95 \\
\hline & & $\mathrm{Vm}$ & 3 & 3 & 3 \\
\hline & & $\mathrm{Me}$ & 6 & 5 & 6 \\
\hline & & VM & 11 & 9 & 11 \\
\hline & & $\mathrm{X}$ & 5,69 & 5,80 & 5,95 \\
\hline & & $S$ & 1,31 & 1,47 & 1,56 \\
\hline & \multirow{6}{*}{ Feminino } & $\mathrm{N}$ & 100 & 100 & 100 \\
\hline & & $\mathrm{Vm}$ & 3 & 3 & 3 \\
\hline & & $\mathrm{Me}$ & 6 & 6 & 6 \\
\hline & & VM & 11 & 10 & 12 \\
\hline & & $x$ & $5,97 \quad(\mathbf{M}=\mathrm{F})$ & $6,16 \quad(M=F)$ & $6,03 \quad(M=F)$ \\
\hline & & $S$ & $1,56 \quad p>0,05$ & $1,30 \quad p>0,05$ & $1,50 \quad p>0,05$ \\
\hline \multirow{12}{*}{ TPC } & \multirow{6}{*}{ Masculino } & $\mathrm{N}$ & 91 & 94 & 95 \\
\hline & & $\mathrm{Vm}$ & 6 & 7 & 7 \\
\hline & & $\mathrm{Me}$ & 12 & 12 & 13 \\
\hline & & VM & 21 & 23 & 30 \\
\hline & & $x$ & 12,26 & 12,53 & 12,79 \\
\hline & & $\mathrm{S}$ & 2,86 & 3,08 & 3,47 \\
\hline & \multirow{6}{*}{ Feminino } & $\mathrm{N}$ & 100 & 100 & 100 \\
\hline & & $\mathrm{Vm}$ & 7 & 8 & 7 \\
\hline & & $\mathrm{Me}$ & 12 & 13 & 13 \\
\hline & & VM & 23 & 24 & 26 \\
\hline & & $x$ & $12,98(M=F)$ & $13,42(\mathbf{M}=\mathrm{F})$ & $13,13(M=F)$ \\
\hline & & $S$ & $3,49 p>0,05$ & $3,05 \quad p>0,05$ & $3,28 p>0,05$ \\
\hline
\end{tabular}

Não houve diferença entre os sexos com relação ao ritmo de piscar, sendo que as meninas e os meninos apresentaram valores bastante semelhantes.

Há informações de que o piscar é virtualmente ausente em bebês e aumenta até a adolescência, permanecendo estável por toda a vida ${ }^{(14)}$.

Entretanto, avaliando-se os dados obtidos no presente estudo segundo a idade da criança, o piscar completo foi menor nas crianças menores ( 4 anos), com tendência ao aumento até a idade de 6 anos. A razão para que as crianças menores apresentassem este padrão é desconhecida.

Esta tendência foi notada quando se avaliou o piscar completo que compreende o tempo de abertura e fechamento completo da pálpebra. Porém, quando se avalia o piscar incompleto que é mais freqüente que o completo, não foi observada diferença estatística entre as idades, tanto no sexo masculino, como no feminino.
O tempo de fechamento foi maior que o tempo de abertura nas crianças, o que também ocorre em adultos ${ }^{(1-2)}$.

Usando metodologia semelhante e avaliando o piscar de indivíduos normais de diferentes idades foi possível notar que o tempo de fechamento palpebral é maior nos idosos ${ }^{(2)}$. Quando se compara o tempo de fechamento palpebral das crianças avaliadas no presente estudo com os dados obtidos naquele estudo $^{(2)}$, observa-se que realmente os idosos gastam mais tempo com o fechamento palpebral que as crianças. Entretanto, o tempo de abertura palpebral é maior nas crianças. Desta forma, a avaliação comparativa do tempo de piscar completo fica semelhante para idosos e crianças e a avaliação apenas deste parâmetro poderia sugerir não haver diferença nas comparações das diferentes idades.

A explicação para se ter maior tempo de fechamento em idosos poderia ser pela redundância de tecidos pela senilida- 


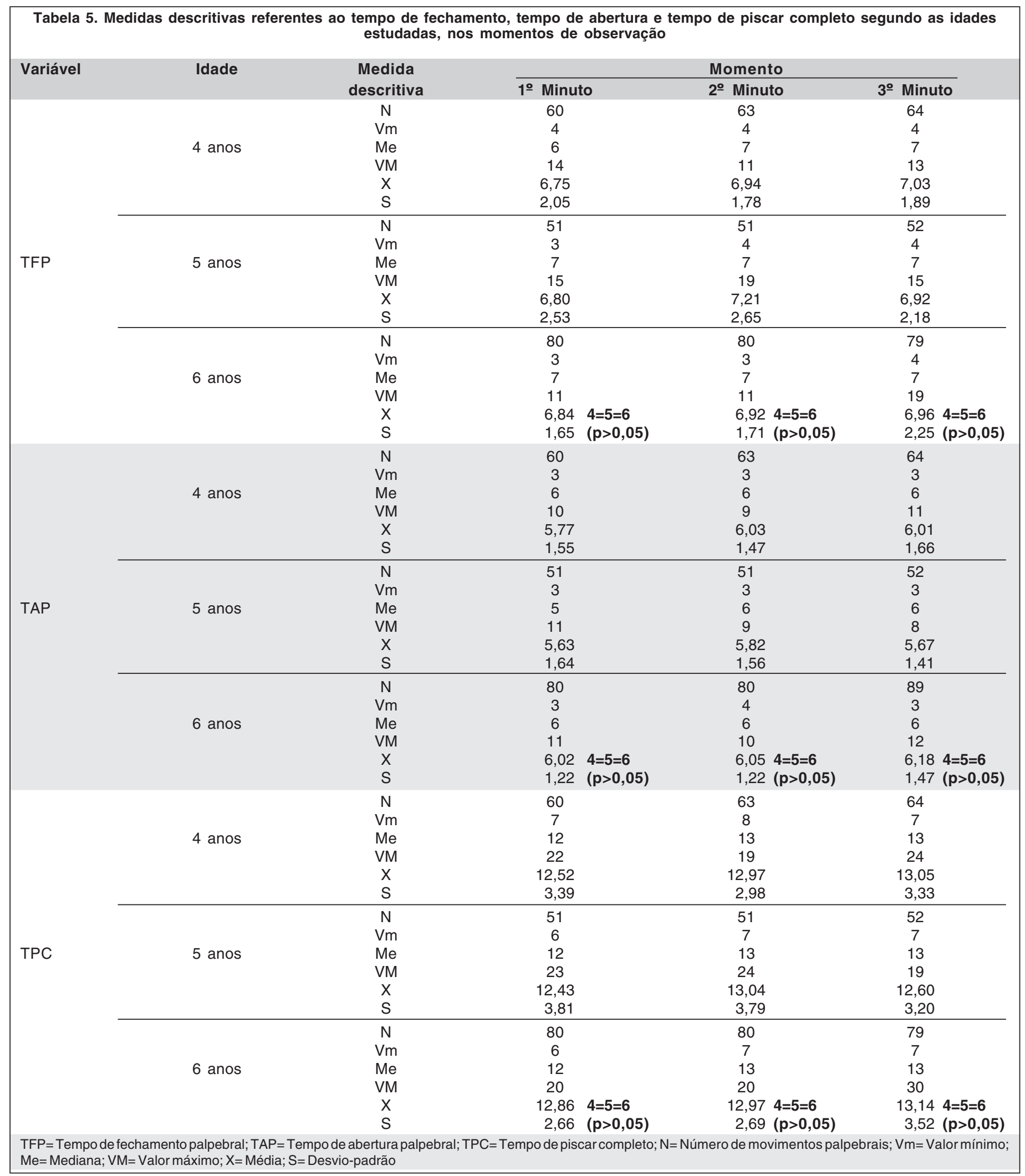

de, com aumento do peso palpebral e conseqüente diminuição da velocidade do movimento palpebral ${ }^{(2)}$.
Desta forma, o presente estudo mostrou que em crianças em idade pré-escolar os tempos de fechamento e de abertura 
palpebral e o tempo de piscar completo foram semelhantes em ambos os sexos, para todas as idades estudadas e em todos os minutos de observação.

Os dados aqui apresentados são, até o momento, inéditos no que toca a obtenção de valores de normalidade para o ritmo de piscar de crianças saudáveis, tendo sido estabelecidos vários parâmetros como ritmo de piscar com relação a gênero, tempo de abertura e fechamento, relação entre piscar completo e incompleto. Desta forma, estabeleceu-se um padrão de normalidade que poderá ser usado em estudos futuros.

\section{CONCLUSÃO}

As crianças apresentam piscar completo mais freqüente que o incompleto. $\mathrm{O}$ ritmo de piscar completo aumenta com a idade. Os tempos de fechamento e de abertura palpebral e o tempo de piscar completo foram semelhantes em ambos os sexos, em todas as idades estudadas, sendo o fechamento mais lento que a abertura palpebral.

\section{ABSTRACT}

Purpose: To evaluate spontaneous blink activity in the primary eye position in normal preschool children. Methods: Two hundred normal children aged 4 to 6 years were prospectively evaluated. They were digitally videotaped in a standard setting, taking the images on frontal and lateral plans, during the vigil state, in the primary eye position. The object of observation was located at the same level of the pupil. A Sony Lithium camera was used. The images were recorded on $8 \mathrm{~mm}$ tapes and transferred to a personal computer (MacIntosh G4) and processed with the iMovie software. Opening, closure and total blink times were measured using frame scale. The eyelid movement rhythm per minute was also evaluated, during three minutes. The data were submitted to statistical analysis. Results: The complete eye blink was more frequently observed than the incomplete eye blink. The blink rate increased with age. The incomplete blink rate was the same for all the evaluated ages. The eyelid opening and closing times and the complete blink time were similar for both sexes, during all evaluation moments. There were no differences between genders regar- ding eye blink. The closing eyelid time was slower than the opening eyelid time. Conclusions: The complete blink rate increases with age. The closing and the opening eyelid times and the complete blink were similar for both sexes in all evaluated ages and the opening time is faster than the closing time.

Keywords: Blinking/physiology; Saccades; Eyelids; Videotape recording; Child, preschool

\section{REFERÊNCIAS}

1. Recordes M. Physicology of the brown and eyelid. In: Tasman W, Jaeger EA, editors. Duane's clinical ophthalmology [CD-ROM]. Philadelphia: JB Lippincott; 1997.

2. Schellini SA, Sampaio AA Jr, Hoyama E, Cruz AA, Padovani CR. Spontaneous eye blink analysis in the normal individual. Orbit. 2005;24(4):239-42.

3. Gordon G. Observations upon the movements of the eyelids. Br J Ophthalmol. 1951;35(6):339-51.

4. Bjork A, Kugelberg E. The electrical activity of the muscles of the eye and eyelids in various positions and during movements. Electroencephal Clin Neurophysiol, Suppl 1953;5(4):595-602.

5. Evinger C, Manning KA, Sibony PA. Eyelid movements. Mechanisms and normal data. Invest Ophthalmol Vis Sci. 1991;32(2):387-400.

6. Fuchs AF, Becker W, Ling L, Langer TP, Kaneko CR. Discharge patterns of levator palpebrae superioris motoneurons during vertical lid and eye movements in the monkey. J Neurophysiol. 1992;68(1):233-43.

7. Porter JD, Stava MW, Gaddie IB, Baker RS. Quantitative analysis of eyelid movement metrics reveals the highly stereotyped nature of monkey blinks. Brain Res. 1993;609(1-2):159-66.

8. Schmidtke K, Büttner-Ennever JA. Nervous control of eyelid function: A review of clinical, experimental and pathological data. Brain. 1992;115 Pt 1:227-47.

9. Sibony PA, Evinger C, Manning KA. Eyelid movements in facial paralysis. Arch Ophthalmol. 1991;109(11):1555-61.

10. Dumery B, Toi VV. Relationship between blink rate, ocular desconfort and visual tasks [abstract]. Invest Ophthalmol Vis Sci. 1997;38(S):68.

11. Sahlin S, Chen E. Gravity, blink rate, and lacrimal drainage capacity. Am J Ophthalmol. 1997;124(6):758-64. Comment in: Am J Ophthalmol. 1998;126 (1): 152 .

12. Tsubota K, Nakamori K. Effects of ocular surface area and blink rate on tear dynamics. Arch Ophthalmol. 1995;113(2):155-8.

13. Barbato G, Ficca G, Muscettola G, Fichele M, Beatrice M, Rinaldi F. Diurnal variation in spontaneous eye-blink rate. Psychiatry Res. 2000;93(2):145-51.

14. Bentivoglio AR, Bressman SB, Cassetta E, Carretta D, Tonali P, Albanese A. Analysis of blink rate patterns in normal subjects. Mov Disord. 1997;12 (6):1028-34.

15. Monster AW, Chan HC, O'Connor D. Long-term trends in human eye blink rate. Biotelem Patient Monit. 1978;5(4):206-22.

16. Caplan R, Guthrie D, Komo S. Blink rate in children with attention-deficithyperactivity disorder. Biol Psychiatry. 1996;39(12):1032-8.

17. Nakamori K, Odawara M, Nakajima T, Mizutani T, Tsubota K. Blinking is controlled primarily by ocular surface conditions. Am J Ophthalmol. 1997; 124(1):24-30

\section{Ao enviar um artigo para publicação,} leia ATENTAMENTE as instruções para autores, constante no final de cada fascículo. 